\title{
Cdk5 Loss Alters Mitochondrial Cristae Organization
}

\author{
Saranya Navaneetha Krishnan', Jesusa L. Rosales', Ki-Young Lee ${ }^{1 *}$ \\ 'Department of Cell Biology \& Anatomy, Arnie Charbonneau Cancer and Alberta Children's Hospital Research Institutes, Cumming School of Medicine, \\ University of Calgary, Calgary, AB, Canada
}

\section{Article Info}

\section{Article Notes}

Received: December 18, 2020

Accepted: January 27, 2021

\section{${ }^{*}$ Correspondence:}

Dr. Ki-Young Lee, Department of Cell Biology and Anatomy, Cumming School of Medicine, University of Calgary, Calgary, AB, T2N 4N1, Canada; Telephone No: (403) 220-8723; Fax No: (403) 283-8727; E-mail: kylee@ucalgary.ca.

(c) 2021 Lee KY. This article is distributed under the terms of the Creative Commons Attribution 4.0 International License.

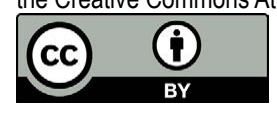

Mitochondria are key cellular organelles executing diverse fundamental functions, including energy conversion, calcium homeostasis, immunity and cell death. These roles are intricately regulated by changes in the structural morphology of the mitochondria ${ }^{1}$. These changes include mitochondrial fusion and fission that are controlled by the multidomain GTPase dynaminrelated proteins (DRPs): mitofusin $1 / 2(\mathrm{Mfn} 1 / 2)$, optic atrophy 1 (Opa1) and dynamin-related proteins 1 (Drp1) ${ }^{1}$. Mfn1/2 and Opa1 promote fusion of adjacent mitochondria while Drp1 stimulates fission. Mfn1/2 is required for the initial tethering of fusing mitochondria ${ }^{2,3}$. Physical interaction of Mfn1/2 with Opa1, which is stabilized by an assembly of OPA1 in the intermembrane space (IMS) ${ }^{4}$, then allows mitochondrial fusion. Conversely, Drp1 causes fission by forming distinct spiral structures on the mitochondrial outer membrane ${ }^{1}$. Mitochondrial fission is also regulated by posttranslational modification of Drp1. Phosphorylation of Drp1 at serine $616\left(\mathrm{Ser}_{616}\right)$ by extracellular signal-regulated kinase 2 (Erk2) promotes fission ${ }^{5}$ while phosphorylation at serine 637 $\left(\mathrm{Ser}_{637}\right)$ by protein kinase A (PKA) inhibits the process ${ }^{6}$. In addition, dephosphorylation of Drp1 at $\operatorname{Ser}_{637}$ by calcineurin stimulates fission ${ }^{7}$.

Cyclin-dependent kinase 5 (Cdk5) is a serine/threonine kinase that was discovered based on its sequence identity to the key cell cycle regulators, $\mathrm{Cdk} 1$ and $\mathrm{Cdk}^{8}{ }^{8}$. However, Cdk5 belongs to the atypical Cdks whose roles are not directly linked to cell cycle control $^{8}$. Recently, Cdk5, a known cytoplasmic protein, was localized to mitochondria, specifically at the inner membrane ${ }^{9}$, and such localization has been associated with a role in regulating mitochondrial dynamics ${ }^{7}$. For example, we recently demonstrated that loss of Cdk5, including mitochondria, causes mitochondrial permeability transition pore (mPTP) opening and subsequent depolarization as well as mitochondrial fission and ROS increase ${ }^{7}$. These events lead to decreased ATP production and ultimately apoptosis in breast cancer cells ${ }^{7}$. Treatment with an mPTP inhibitor, cyclosporine A or sanglifehrin A, reverses the phenotypic effects, including breast cancer cell apoptosis, due to Cdk5 loss ${ }^{7}$, supporting a role for $\mathrm{Cdk} 5$ in regulating mPTP dynamics.

Maintenance of structural integrity and balance between fusion and fission states are crucial for proper mitochondrial function; perturbation of which could trigger apoptosis. Increased fission resulting from DRP1 translocation into mitochondria and oligomerization at the fission site, causing membrane ingression, is an early apoptotic event $t^{1}$ that is influenced by increased intracellular 
calcium concentration $\left(\left[\mathrm{Ca}^{2+}\right]_{\mathrm{i}}\right)^{7}$. In breast cancer cells, we found that $\mathrm{Cdk} 5$ loss causes elevated $\left[\mathrm{Ca}^{2+}\right]_{i}$ that coincides with a surge in $\mathrm{Ca}^{2+}$-dependent calcineurin activity and subsequent dephosphorylation of DRP1 at $\mathrm{Ser}_{637}$ as well as mitochondrial fission ${ }^{7}$. These findings suggest that Cdk5 loss promotes breast cancer cell apoptosis by inducing mitochondrial fission via the $\left[\mathrm{Ca}^{2+}\right]_{i}$-calcineurin-DRP1

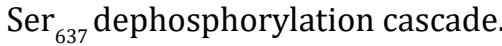

Cristae, the oxidative phosphorylation site in mitochondria, are deep invaginations that result from disruption in the continuity of the inner mitochondrial membrane (IMM). The IMM is squeezed at its base into narrow openings called cristae junctions, which prevent diffusion of cristae contents such as cytochrome $\mathrm{c}$ into the IMS. Indeed, the release of cytochrome $c$ into the cytoplasm is an early apoptotic event triggered by IMM ultrastructure dynamics or cristae remodelling. However, it was reported that mPTP also regulates cristae junction remodelling for cytochrome c-dependent apoptosis induced by ER stress $^{10}$. In previous studies, we further found that loss of Cdk5, which regulates mPTP dynamics ${ }^{7}$, results in caspase activation ${ }^{7}$ that is mediated by cytochrome c release, further supporting a role for Cdk5 in mitochondria-mediated apoptosis, and potentially, in cristae organisation.

Opa1 controls cristae integrity independently of its role in mitochondrial fusion ${ }^{11}$. In humans, eight alternatively spliced variant forms of Opa1 encode for proteins that contain $\mathrm{N}$-terminal mitochondria targeting sequence, a transmembrane and a coiled-coil domain ${ }^{11}$. Cleavage of the mitochondrial targeting sequence generates the membrane-anchored Opa1 long forms (L-forms) that are further cleaved at their $\mathrm{N}$ terminus by Opa1 proteases,

A

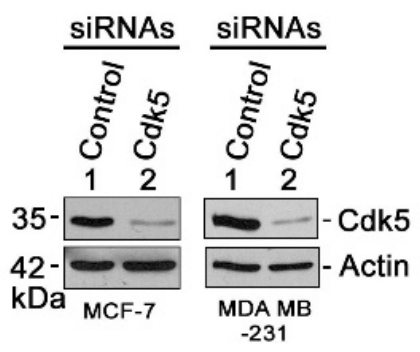

B
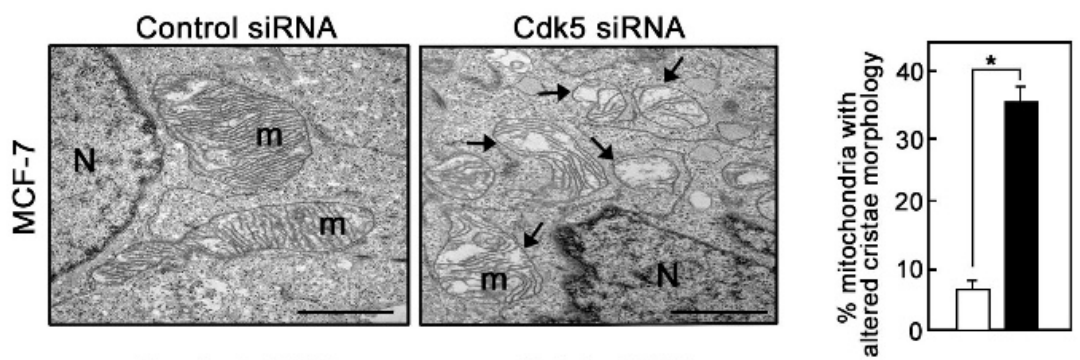

C
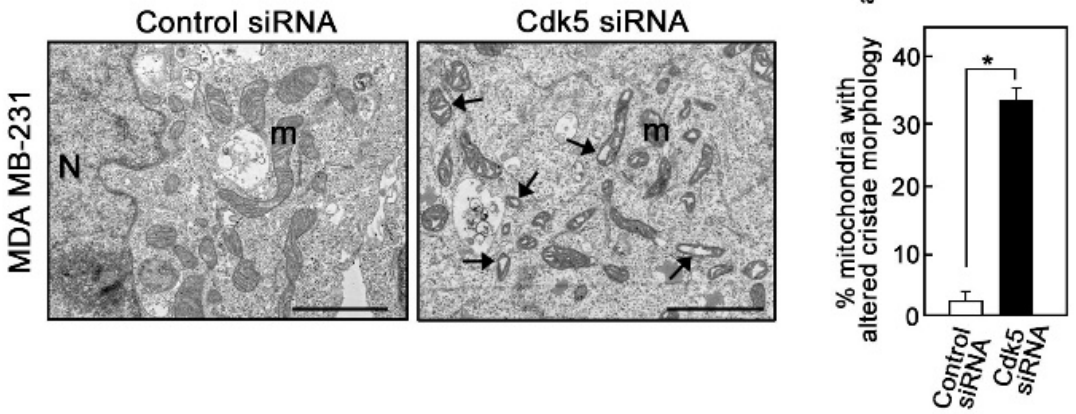

Figure 1. Loss of Cdk5 causes mitochondrial cristae disorganization in breast cancer cells. A. MCF-7 and MDA MB-231 breast cancer cells transfected with Cdk5 siRNA (100 nM) for $72 \mathrm{hrs}$ were lysed and subjected to immunoblotting for Cdk5. Actin blot serves as loading control. Blots shown represent one of three sets of blots with similar patterns. B and C. Transmission electron microscopy (TEM) images of mitochondrial ultrastructure in MCF-7 (B) and MDA MB-231 (C) cells transfected with control or Cdk5 siRNA. Images were acquired using Hitachi H7650 TEM at 7,000x magnification. Scale bar $=500 \mathrm{~nm}$. Arrows in Cdk5-depleted cells are directed at mitochondria (m) with altered cristae structure. N represents nucleus. Graphs show the \% of mitochondria with clearly disorganized cristae structure. Data were acquired by blindly analyzing 50 mitochondria per treatment group in each of three independent experiments. Values are means \pm SEM from the three independent experiments. ${ }^{*}$ indicates $p<0.05$ using student's t-test (unpaired). 
generating the soluble short forms (S-forms) in the IMS $^{11}$. Oligomerization of S- and L-forms in the IMS and the IMM, respectively, regulates the formation of cristae junctions. Previously, it was shown that Cdk5 interacts with and phosphorylates $0 \mathrm{mi} / \mathrm{HtrA2}{ }^{9}$, an 0 pa1 protease $^{12}$. Specific phosphorylation of Omi/HtrA2 at $\mathrm{Ser}_{400}$ by Cdk5 elevates its protease activity ${ }^{9}$, increasing Opa1 cleavage and thus cristae junction formation ${ }^{12}$. Indeed, defects in Opa1 processing have been shown to cause disorganized cristae structure $^{13}$. Since OPA1 is also cleaved by OMA1 and Yme1L, it would be interesting to examine whether loss of Cdk5 affects the activity of OMA1 and Yme1L.

Cristae organisation is also regulated by $\left[\mathrm{Ca}^{2+}\right]_{i}^{14}$. It is interesting that depletion of OPA1 was shown to increase the rate of mitochondrial $\mathrm{Ca}^{2+}$ uptake, indicating the importance of OPA1 in regulating mitochondrial $\mathrm{Ca}^{2+}$ dynamics ${ }^{15}$, and suggesting a role for $\left[\mathrm{Ca}^{2+}\right]_{\mathrm{mt}}$ in controlling OPA1-mediated cristae organisation. In separate studies, $\mathrm{Ca}^{2+}$ transfer from the endoplasmic reticulum (ER) to the mitochondria and subsequent activation of MPTP was also found to contribute to cristae remodelling ${ }^{16}$. Thus, our observation that Cdk5 loss in primary mouse embryonic fibroblasts (MEFs) causes increased $\mathrm{Ca}^{2+}$ transfer from the
ER to the mitochondria, resulting in increased $\left[\mathrm{Ca}^{2+}\right]_{\mathrm{mt}}$ and mPTP opening ${ }^{17}$ further supports a link between Cdk5 and cristae organisation.

Here, we sought to examine whether Cdk5 loss triggers a change in IMM topology and affects cristae structural organization. As shown in Figure 1, Cdk5 depletion by siRNA in two representative breast cancer cell lines, MCF7 and MDA MB-231, and subsequent electron microscopy revealed that while cells transfected with control siRNA displayed normal tubular mitochondria with wellorganized cristae, cells depleted of Cdk5 showed irregular cristae structures with increased spaces between adjacent cristae. This dramatic alteration in cristae structure in breast cancer cells depleted of Cdk5 was recapitulated in ex vivo MEFs from Cdk5 ${ }^{-}$mice (Figure 2). Altogether, our results indicate a novel role for Cdk5 is maintaining mitochondrial cristae integrity and thus loss of Cdk5 causes cristae disorganization. Since Cdk5 phosphorylates Omi/HtrA2 at $\mathrm{Ser}_{400}{ }^{9}$, increasing its protease activity ${ }^{9}$ and Opa1 cleavage ${ }^{12}$, it is possible that loss of Cdk5 results in loss of Omi/HtrA2 $\mathrm{Ser}_{400}$ phosphorylation and reduced Opa1 cleavage, leading to disorganized cristae structure. Since Cdk5 further regulates $\left[\mathrm{Ca}^{2+}\right]_{\mathrm{mt}}$ and mPTP dynamics,

A

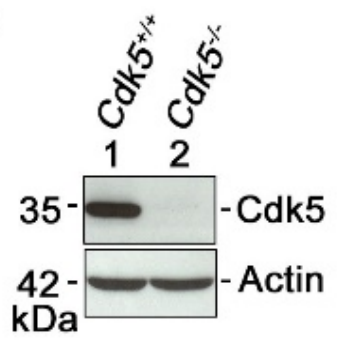

B


Figure 2. Cdk5 $\%$ primary mouse embryonic fibroblast cells exhibit disorganized cristae structure. A. Lysates of wt and Cdk5 $\%$ MEFs isolated from wt and Cdk5 $\%$ E13.5 embryos were subjected to SDS-PAGE and immunoblotting for Cdk5. Actin blot serves as loading control. Blots shown represent one of three sets of blots with similar patterns. B. TEM of mitochondrial ultrastructure in wt and $\mathrm{Cdk5} \% \mathrm{MEFs}$. Arrows in Cdk5\% MEFs are directed at mitochondria with disorganized cristae. Images were acquired using Hitachi H7650 TEM at 10,000x magnification. Scale bar $=500 \mathrm{~nm}$. Graph shows the \% of mitochondria $(\mathrm{m})$ with clearly disorganized cristae structure. Analysis was performed blindly in ten different fields per treatment group. Values represent means \pm SEM from three independent experiments. ${ }^{*}$ indicates $p<0.05$ using student's t-test (unpaired). 
which have both been implicated in cristae organization, it is also possible that altered $\left[\mathrm{Ca}^{2+}\right]_{\mathrm{mt}}$ or $\mathrm{mPTP}$ opening led to cristae disorganization upon loss of Cdk5. However, a combined effect of Cdk5 loss on Omi/HtrA2 $\mathrm{Ser}_{400}$ phosphorylation, $\left[\mathrm{Ca}^{2+}\right]_{\mathrm{m},}$, and mPTP dynamics likely causes cristae disorganization.

\section{Acknowledgement}

This work was supported by a grant from NSERC (RGPIN/06270-2019) to KYL.

\section{References}

1. Westermann B. Mitochondrial fusion and fission in cell life and death. Nat Rev Mol Cell Biol 2010; 11:872-84.

2. Ishihara N, Eura Y, Mihara K. Mitofusin 1 and 2 play distinct roles in mitochondrial fusion reactions via GTPase activity. J Cell Sci 2004; 117:6535-46.

3. Koshiba T, Detmer SA, Kaiser JT et al. Structural basis of mitochondrial tethering by mitofusin complexes. Science 2004; 305:858-62.

4. Detmer SA, Chan DC. Functions and dysfunctions of mitochondrial dynamics. Nat Rev Mol Cell Biol 2007; 8:870-9.

5. Kashatus JA, Nascimento A, Myers LJ et al. Erk2 phosphorylation of Drp1 promotes mitochondrial fission and MAPK-driven tumor growth. Mol Cell 2015; 57:537-51.

6. Cribbs JT, Strack S. Reversible phosphorylation of Drp1 by cyclic AMPdependent protein kinase and calcineurin regulates mitochondrial fission and cell death. EMBO Rep 2007; 8:939-44.

7. Navaneetha Krishnan S, Rosales JL, Lee KY. Loss of Cdk5 in breast cancer cells promotes ROS-mediated cell death through dysregulation of the mitochondrial permeability transition pore. Oncogene 2018.
8. Rosales JL, Lee KY. Extraneuronal roles of cyclin-dependent kinase 5. Bioessays 2006; 28:1023-34.

9. Fitzgerald JC, Camprubi MD, Dunn L et al. Plun-Favreau H. Phosphorylation of HtrA2 by cyclin-dependent kinase-5 is important for mitochondrial function. Cell Death Differ 2012; 19:257-66.

10. Zhang D, Lu C, Whiteman M et al. The mitochondrial permeability transition regulates cytochrome $\mathrm{c}$ release for apoptosis during endoplasmic reticulum stress by remodeling the cristae junction. J Biol Chem 2008; 283:3476-86

11. Del Dotto V, Fogazza M, Carelli V et al. Eight human OPA1 isoforms, long and short: What are they for? Biochim Biophys Acta 2018; 1859:263-9.

12. Kieper N, Holmstrom KM, Ciceri D et al. Modulation of mitochondrial function and morphology by interaction of 0mi/HtrA2 with the mitochondrial fusion factor OPA1. Exp Cell Res 2010; 316:1213-24.

13. Hering T, Kojer K, Birth $\mathrm{N}$ et al. Mitochondrial cristae remodelling is associated with disrupted OPA1 oligomerisation in the Huntington's disease R6/2 fragment model. Exp Neurol 2017; 288:167-75.

14. Gottschalk B, Klec C, Waldeck-Weiermair $M$ et al. Intracellular $\mathrm{Ca}(2+)$ release decelerates mitochondrial cristae dynamics within the junctions to the endoplasmic reticulum. Pflugers Arch 2018; 470:1193-203.

15. Fulop L, Szanda G, Enyedi B et al. The effect of OPA1 on mitochondrial $\mathrm{Ca}(2)(+)$ signaling. PLoS One 2011; 6:e25199.

16. Germain M, Mathai JP, McBride HM, Shore GC. Endoplasmic reticulum BIK initiates DRP1-regulated remodelling of mitochondrial cristae during apoptosis. EMBO J 2005; 24:1546-56.

17. Navaneetha Krishnan S, Rosales JL, Lee KY. mPTP opening caused by $\mathrm{Cdk} 5$ loss is due to increased mitochondrial $\mathrm{Ca}(2+)$ uptake. Oncogene 2020; 39:2797-806. 